\title{
Cultura organizacional para manejo y prevención de riesgos laborales en una institución educativa, Quito-Ecuador
}

\author{
Organizational culture for management and prevention of occupational \\ risks in an educational institution, Quito-Ecuador \\ Cultura organizacional para gestão e prevenção de riscos ocupacionais em uma \\ instituição de ensino, Quito-Equador
}

\author{
Sergio Naranjo Herrera \\ sergio.naranjo@cordillera.edu.ec \\ https://orcid.org/0000-0003-1782-7656
}

\author{
Sara Vásquez Coba \\ sara.vasquez@cordillera.edu.ec \\ https://orcid.org/0000-0003-0445-9364
}

\section{Instituto Tecnológico Universitario Cordillera, Quito-Ecuador}

\section{RESUMEN}

Las enfermedades ocupacionales $y$ relacionadas con la actividad laboral y el ausentismo son factores que dependen de las condiciones de los entornos laborales de colaboradores de las instituciones. La investigación tuvo por objetivo analizar los riesgos reportados en el personal de una institución de educación superior. En el 2019 se registraron alrededor de 831 atenciones médicas relacionadas a distintas patologías, las más comunes fueron las enfermedades musculoesqueléticas, respiratorias, metabólicas y neurológicas. El ausentismo incide en el incremento en costos económicos y en el desempeño laboral, se estimó que el impacto en horas perdidas debidas a los permisos médicos fue de 4093 horas, sobre las cuales, el médico ocupacional generó 349 horas de ausentismo que corresponde al $9 \%$ de horas perdidas. Dentro de los diagnósticos por enfermedad los más numerosos están la epicondilitis lateral, trastorno mixto de ansiedad y depresión, cervicalgia, síndrome cérvico-branquial y lumbago no específico. La planeación, control y vigilancia garantizan sitios de trabajo seguros, sanos y el bienestar físico, mental y social de cada persona.

Palabras clave: Riesgo psicosocial; Ausentismo laboral; Psicología de la salud ocupacional; Músculo-esquelético; Responsabilidad social en salud

\section{ABSTRACT}

Occupational and work-related diseases and absenteeism are factors that depend on the conditions of the work environments of collaborators of the institutions. The research aimed to analyze the risks reported in the staff of a higher education institution. In 2019, about 831 medical attentions related to different pathologies were recorded, the most common were musculoskeletal, respiratory, metabolic and neurological diseases. Absenteeism has an impact on the increase in economic costs and work performance, it was estimated that the impact on hours lost due to medical leave was 4093 hours, of which the occupational physician generated 349 hours of absenteeism, which corresponds to $9 \%$ of hours lost. Among the diagnoses due to illness, the most numerous are lateral epicondylitis, mixed anxiety and depression disorder, cervicalgia, cervico-branchial syndrome and nonspecific lumbago. Planning, control and surveillance guarantee safe, healthy workplaces and the physical, mental and social well-being of each person.

Key words: Psychosocial risk; Work absenteeism; Occupational health psychology; Musculoskeletal; Social responsibility in health

\section{RESUMO}

Doenças ocupacionais e relacionadas ao trabalho e absenteísmo são fatores que dependem das condições dos ambientes de trabalho dos funcionários nas instituições. A pesquisa visava analisar os riscos relatados no pessoal de uma instituição de ensino superior. Em 2019, foram registradas cerca de 831 atenções médicas relacionadas a diferentes patologias, sendo as mais comuns as doenças músculo-esqueléticas, respiratórias, metabólicas e neurológicas. 0 absenteísmo tem um impacto no aumento dos custos econômicos e no desempenho do trabalho. Foi estimado que o impacto nas horas perdidas devido à licença médica foi de 4093 horas, das quais o médico do trabalho gerou 349 horas de absenteísmo, o que corresponde a $9 \%$ das horas perdidas. Entre os diagnósticos devidos a doenças, os mais numerosos são epicondilites laterais, distúrbios mistos de ansiedade e depressão, cervicalgia, síndrome cervico-branquial e lumbago não específico. O planejamento, controle e monitoramento garantem locais de trabalho seguros e saudáveis e o bem-estar físico, mental e social de cada indivíduo.

Palavras-chave: Risco psicossocial; Absenteísmo; Psicologia da saúde ocupacional; Musculo-esquelético; Responsabilidade social em saúde 


\section{INTRODUCCIÓN}

L os empleados de una institución están expuestos a entornos laborales y condiciones de trabajo específicas a su puesto de trabajo y entorno laboral que deben ser

evaluadas y podrían, en algún punto relacionarse con enfermedades del trabajo. Los problemas de salud, resultado de una afectación física o emocional de un trabajador repercuten en la calidad de vida, lo que supone un costo social, económico y productivo que se debe considerar cada año en una empresa/institución.

Es necesario aclarar que la salud no hace referencia a la "ausencia de enfermedad o afección" sino, como la Organización Mundial de la Salud la define es "un estado de bienestar físico, mental y social (bienestar integral)" (OMS, 2020). Alonso et al. (2004) describe en sus investigaciones que algunas de las causas más comunes de factores psicosociales reportadas son el género, la menor edad, el nivel educativo más bajo, el nivel de urbanidad más alto, no vivir con acompañante y situaciones de desempleo.

La psicología de la salud ocupacional (PSOP), constituye un marco teórico nuevo que rompe, de cierta manera, con el esquema de mirar a la psicología como la ciencia del mal funcionamiento de las personas en distintos ámbitos. Esta perspectiva teórica positiva se enfoca en la mejora de la calidad de vida laboral y organizacional mediante la introducción de los conceptos de bienestar y la satisfacción de los trabajadores y el conocimiento de la "vida organizacional positiva" (Salanova et al., 2016). La psicología de la salud ocupacional se enmarca en mejorar de la calidad de vida laboral y a proteger la seguridad, la salud y bienestar de los empleados (Sociedad Venezolana de Psicología Positiva, 2014; Salanova, 2008).

Sin duda, toda institución busca el crecimiento organizacional que constituye un pilar fundamental en el sostenimiento de una empresa. El recurso humano es la clave para que la administración de una institución tenga éxito; el talento humano, sus capacidades y habilidades son la pieza fundamental para el funcionamiento eficiente (Soler et al., 2019). Es así que, el objetivo de toda organización es la mejora del desempeño organizacional que incluye la mejora de la calidad de vida del personal que contiene tres aspectos esenciales: la salud psicosocial, el bienestar y la satisfacción de los trabajadores

La definición de los factores psicosociales en el trabajo (antes llamados riesgos) según la Organización Internacional del Trabajo (OIT, 1984; OIT, 2020) consiste: "en interacciones entre el trabajo, su medio ambiente, la satisfacción en el trabajo y las condiciones de su organización, por una parte".

La promoción de la seguridad e higiene de los puestos de trabajo no solo debe tomar en cuenta los factores físicos, químicos, mecánicos y biológicos del medio ambiente sino también los psicosociales que pueden afectar al bienestar integral de la persona en los 
ámbitos físicos y mentales. Los factores de riesgo psicosocial son numerosos y dependen de los puestos de trabajo.

Cada organización desarrolla diversas estrategias para enfrentar los cambios y exigencias y de los organismos de control en cuanto al manejo de situaciones de riesgo y a la prevención de los mismos. Disponer de metodologías sencillas de evaluación de riesgos laborales en una institución es una prioridad para su control. El riesgo se determina de acuerdo a dos factores que son la probabilidad y la magnitud de los daños (consecuencias). Existen varias repercusiones en las personas que sufren un accidente de trabajo o enfermedad profesional. Esta situación no solo afecta a la planta de trabajadores sino a sus familias en su bienestar físico, emocional y, en algunas ocasiones, en el aspecto económico.

Las condiciones de trabajo, las capacidades y necesidades del personal de una institución determinan la interacción del personal y su ambiente de trabajo (OIT, 1984). El riesgo laboral, más allá de su definición como, la probabilidad a la exposición de un factor ambiental peligroso, influye en las condiciones de trabajo y el bienestar del trabajador. Dentro de los riesgos, se describen los de índole ergonómica y los psicosociales, sobre los cuales se discutirá en esta investigación. Estos dos tipos dependen directamente de las demandas organizaciones y personales. Según Gil (2014, p. 30) se definen los riesgos psicosociales como "los aspectos del diseño del trabajo, de la organización y de la dirección del trabajo, y sus contextos sociales y organizacionales, que tienen el potencial de causar un daño psicológico, físico o social al individuo".

Elausentismo laboral constituye un problema común en las institucionesyla identificación de las causas y su seguimiento resulta un reto grande para las empresas. Las ausencias, en la mayoría de casos, se asocian a una exposición a riesgos laborales, tanto psicosociales como ergonómicos, y a su vez a factores externos propios de la actividad y cargo en la institución.

La meta de una organización en conformarse y mantenerse como una Organización saludable (OS), que significa que el modelo organizacional debe velar por el cuidado de sus empleados. La salud psicosocial de los empleados es el pilar para lograr los objetivos institucionales y otros fines estratégicos intrínsecos. El concepto de OS es una visión integradora de varios conceptos y disciplinas y especiales que incluyen estudios de estrés ocupacional, seguridad y salud ocupacional, promoción de la salud y conducta organizacional en los departamentos de desarrollo del talento humano y financiero de la institución (Salanova et al., 2016).

Por esta razón, es importante la implementación de medidas correctivas y preventivas dentro de la gestión organizacional de una institución. En ese sentido, el objetivo de la investigación fue evaluar los psicosociales de los puestos de trabajo y relacionarlos con el ausentismo reportado en los empleados de una IES de Quito, Ecuador en el año 2019. 


\section{MÉTODO}

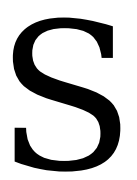

e realizó un estudio descriptivo usando la metodología sobre la evaluación de riesgos definida como la acción prioritaria para una institución. En este estudio se ha aplicado el enfoque precautorio para evitar el control de los efectos y la prevención entre ellos. Este enfoque se basa en 5 pilares específicos que son: la responsabilidad, el respeto, prevención, obligación y la información (Riechmann y Tickener, 2002) que incluyó el análisis de riesgos laborales; registro de atenciones médicas; morbilidad general, y análisis de ausentismo.

Para la evaluación de riesgo laboral, se aplicó el cuestionario validado sobre Evaluación de riesgos psicosociales tomado del Instituto Nacional de Seguridad e higiene en el Trabajo de España. Este cuestionario fue aplicado a 172 personas que pertenecen a 60 puestos de trabajo y sus resultados fueron codificados con el fin de velar por la confidencialidad de los participantes. El cuestionario incluyó 44 preguntas con escala Likert con 3 y 4 opciones de respuesta que se basan en las fases de la Nota técnica de Prevención (NTP) número 702 editada por el Instituto Nacional de Seguridad e Higiene en el Trabajo -INSHT- (INSHT, 2015).

Existen varias orientaciones para evaluar los factores de riesgo psicosocial que sigue seis fases: 1) Identificación de los factores de riesgo; 2) Elección de la metodología, técnicas e instrumentos que se han de aplicar; 3) planificación y realización de trabajo de campo; 4) Análisis de los resultados y elaboración de informe; 5) Elaboración y puesta en marcha de un programa de intervención; 6) seguimiento y control de las medidas adoptadas. Como parte de la metodología se tomaron las cuatro primeras fases para evaluar los factores de riesgo psicosocial. Se realizó una matriz de cumplimiento legal, basada en la norma técnica 330 del sistema simplificado de accidentes de trabajo. Se describieron los factores de riesgo laboral identificados a través del análisis de la matriz de riesgos a través del análisis de 60 puestos de trabajo que se clasificaron en puestos clave, estratégicos y de soporte. La población fue de 172 personas que incluyó a: 94 mujeres, 78 hombres y 5 personas con capacidades especiales. Los factores de riesgos identificados fueron (Tabla 1 ).

Tabla 1. Factores de riesgo laboral identificados en la población de estudio.

\section{FACTOR DE RIESGO}

Agresión o maltrato (verbal o físico)

Alta responsabilidad

Amenaza delincuencial

Atropello o golpe con vehículo

Caída de personas a distinto nivel 


\section{FACTOR DE RIESGO}

Caída de personas al mismo nivel

Calidad de aire interior

Choque contra objetos inmóviles

Choque contra objetos móviles

Confort térmico

Contactos eléctricos indirectos

Contaminantes biológicos

Déficit en la comunicación

Desmotivación

Exposición a radiación solar

Inadecuada supervisión

Inestabilidad emocional

Inestabilidad en el empleo

Inmersión en líquidos o material particulado

Manipulación de cargas

Minuciosidad de la tarea

Movimientos Repetitivos

Posiciones forzadas

Puesto de trabajo con Pantalla de Visualización de Datos (PVD)

Radiación no ionizante

Relaciones interpersonales inadecuadas o deterioradas

Ruido

Sobrecarga mental

Superficies irregulares

Temperatura Ambiente

Trabajo a presión

Trato con clientes y usuarios

Estos riesgos fueron evaluados con la escala de nivel de intervención (Tabla 2), para ello fueron evaluados individualmente los niveles de riesgo por cada puesto de trabajo de acuerdo al nivel de deficiencia, nivel de exposición, nivel de probabilidad, nivel de consecuencia. 
Tabla 2. Escala de evaluación de nivel de riesgo y escala de nivel de intervención.

\begin{tabular}{cccc}
\hline & NIVEL DE RIESGO & NIVEL DE INTERVENCIÓN \\
\hline B & Riesgo bajo & Nivel de intervención III intervención IV & $\begin{array}{c}\text { No intervenir salvo que } \\
\text { un análisis más preciso lo } \\
\text { justifique } \\
\text { Mejorar si es posible. } \\
\text { Justificar su intervención } \\
\text { y rentabilidad }\end{array}$ \\
A & Riesgo moderado & Nivel de intervención II & $\begin{array}{c}\text { Corregir y adoptar } \\
\text { medidas de control } \\
\text { Situación crítica } \\
\text { corrección urgente }\end{array}$ \\
\hline
\end{tabular}

Se calcularon los estadísticos descriptivos de la población y se tabularon los datos registrados en las encuestas en línea realizadas a los empleados de la Institución. Además, se realizó un registro diario de las atenciones médicas realizadas en el año 2019 en donde se registraron 831 casos con detalle por: sexo, edad, condición especial. Además, se diferenció el tipo de atención médica por los siguientes motivos: enfermedad común, enfermedad laboral, enfermedad ocupacional.

Se analizó la morbilidad general del personal institucional en el año 2019. Se realizaron 831 atenciones al personal que se encuentra en relación de dependencia.

Como resultado del análisis de las causas del ausentismo laboral de la institución, la naturaleza del permiso médico (particular o público) que fueron validadas por la institución que regula esta autorización a nivel nacional ecuatoriano (Instituto Ecuatoriano de Seguridad Social, IESS). A más del reporte de ausentismo, se registraron las atenciones médicas, número de certificados médicos y de reposo.

\section{RESULTADOS}

\footnotetext{
continuación, se analizaron 27 factores de riesgo que fueron categorizados como: I- Situación crítica corrección urgente; II- Corregir y adoptar medidas de control; 1 III- Mejorar si es posible. Justificar su intervención y rentabilidad; IV - No intervenir salvo que un análisis más preciso lo justifique. La Tabla 3 detalla los factores más comunes que estuvieron en la primera categoría dentro de los 60 puestos de trabajo.
} 
Tabla 3. Lista de factores críticos reportados que requieren una corrección urgente en varios puestos de trabajo.

\section{Factores críticos de corrección urgente}

Movimientos Repetitivos

Amenaza delincuencial

Posiciones forzadas

Puesto de trabajo con Pantalla de Visualización de Datos (PVD)

Contaminantes biológicos

Movimientos Repetitivos

Se registraron 853 casos de atenciones médicas totales en el año 2019, éstas incluyeron: atenciones curativas relacionadas con el trabajo y atenciones médicas preventivas.

Se obtuvieron un promedio de 77 casos por mes en los que mayoritariamente las atenciones fueron a pacientes mujeres (61\%) y el resto a hombres. El rango de edad de mayor atención fue de 36-49 años, seguido de el de 20-35 y luego los mayores a 50 años. Muchas de las demandas de atención fueron de embarazadas y en menor medida los que presentan capacidades especiales.

Dentro de las 10 primeras causas se aprecian que el $18,5 \%$ las representan los procesos músculo-esqueléticos (entre los que más se evidencian son inflamación de los codos o epicondilitis y las cervicalgia), seguidos por el 17,7\% de los casos por problemas respiratorios (en mayor medida los asociados a las vías respiratorias altas); se presenta un $11,4 \%$ por patologías de orden metabólico (principalmente las hiperlipidemias) y porcentajes menores las Gastroenterológicas, diagnósticos neurológicos, infecciones, otorrinolaringológicas, traumáticas, ginecológicas y psicológicas. La tabla 4 detalla los diagnósticos más comunes en las dos primeras categorías de patologías.

Tabla 4. Diagnósticos de procesos músculo-esqueléticos y respiratorios en el 2019.

\begin{tabular}{cc}
\hline \multicolumn{1}{c}{ Codificación CIE 10 } & \multicolumn{1}{c}{ Diagnósticos } \\
\hline M531 & Procesos músculo-esqueléticos \\
M542 & Síndrome cervicobraquial \\
M545 & Cervicalgia \\
M624 & Lumbago no especificado \\
M771 & Contractura muscular \\
\hline
\end{tabular}




\begin{tabular}{|c|c|}
\hline Codificación CIE 10 & Diagnósticos \\
\hline \multicolumn{2}{|c|}{ Patologías respiratorias } \\
\hline J00 & Rinofaringitis aguda (resfriado común) \\
\hline J029 & Faringitis aguda, no especificada \\
\hline J040 & Laringitis aguda \\
\hline J019 & Sinusitis aguda, no especificada \\
\hline$J 039$ & Amigdalitis aguda, no especificada \\
\hline$J 300$ & Rinitis vasomotora \\
\hline$J 209$ & Bronquitis aguda, no especificada \\
\hline $\mathrm{J} 370$ & Laringitis crónica \\
\hline J042 & Laringotraqueitis aguda \\
\hline$J 304$ & Rinitis alérgica, no especificada \\
\hline $\mathrm{J} 410$ & Bronquitis crónica no especificada \\
\hline R060 & Disnea \\
\hline $\mathrm{J} 110$ & Influenza con neumonía, virus no identificado \\
\hline J060 & Laringofaringitis aguda \\
\hline$J 189$ & Neumonía, no especificada \\
\hline J018 & Otras sinusitis agudas \\
\hline J041 & Traqueitis aguda \\
\hline
\end{tabular}

Durante la investigación se realizó el reporte de los riesgos laborales totales de la población analizada. En la población de 172 personas describieron casos con vulnerabilidades relacionadas a distintas causas de índole física (osteo-muscular), enfermedades metabólicas, endocrinológicas y diagnóstico de tipo psicológico codificados por El Código Internacional de Enfermedades -CIE10-. El 15\% de las causas de los riesgos son las osteomusculares que se encuentran relacionadas al riesgo ergonómico reportadas en la Tabla 4.

Algunos de los factores protectores detectados en la organización fueron: compromiso organizacional, convivencia con la familia nuclear de la mayoría de colaboradores, inclusión del personal en planes de intervención, beneficios institucionales indirectos mediante convenios interinstitucionales y pertenencia a redes de apoyo interna para detección de problemas a tiempo.

Como parte de las acciones preventivas institucionales se realizan constantes seguimientos de casos médicos dentro de la planta de colaboradores con el fin de focalizar los esfuerzos en intervenciones de bienestar integral a grupos puntuales con riesgos laborales a través de redes de apoyo por unidades académico-administrativas. Este último aspecto es una estrategia institucional que se encuentra en mejoramiento constante y análisis para 
poder mantener los índices de incidencias médicas y ocupacionales en los valores más bajos por año.

\section{Discusión}

El análisis de los riesgos psicosociales de instituciones educativas tiene mucha relevancia para la prevención de trastornos mentales y físicos a mediano y largo plazo del personal colaborador. La prevalencia de afecciones emocionales y de trastornos de salud mental es multifactorial; algunos de los factores relacionados y reportados en la literatura son sociales, culturales, ambientales y económicos (WHO, 2020). Los problemas de salud tienen relación significativa con acontecimientos estresantes personales o laborales que puedan estallar un episodio depresivo o de ansiedad en una persona.

La unidad de análisis de la investigación fue el puesto de trabajo agrupado por tres criterios: la gestión con las personas, el margen de autonomía a la hora de realizar la tarea y su naturaleza (INSST, 2015). Por un lado, en las patologías que se relacionan con los factores de riesgo se puede determinar que las causas músculo esqueléticas, mayormente dependen de condiciones ergonómicas y mecánicas en algunos cargos. Algunos factores como el peso excesivo o manifestaciones neurológicas pueden estar relacionados a factores relacionados a la exposición a pantallas de visualización de datos -PDV-. Las afecciones gastrointestinales podrían estar relacionadas al riesgo psicosocial; y las patologías como las otorrinolaringológicas y respiratorias que pueden estar relacionados a factores del riesgo físico/biológico. En las mujeres el aumento de las lesiones osteomusculares puede deberse al posible desarrollo de mayor número de actividades extra laborales (domésticas) de esta población. Además, existe una incipiente cultura preventiva por parte de la población analizada para someterse a controles médicos periódicos desde temprana edad.

Por otro lado, existen las afectaciones emocionales, en donde, se reportó que las mujeres tienen una mayor ocurrencia de estos impactos emocionales pueden llevarlas a la somatización de los mismos con patologías físicas o trastornos de salud mental. Las mujeres son más propensas a sufrir afecciones emocionales causadas por ansiedad que los hombres. Las preocupaciones y miedos recurrentes pueden llegar a afectar la salud mental (Barra et al., 2005). Las personas pueden llegar a tener traumas físicos o emocionales al experimentar una situación de estrés; las exigencias administrativas o laborales según el tipo de puesto de trabajo varían de una persona a otra y sus impactos también. Quienes presentan trastornos de ansiedad pueden aminorar o sobrellevar los síntomas a través de un tratamiento (psicoterapia) adecuada por un tiempo (APA, 2010); en ese sentido, se han realizado convenios específicos con instituciones para que los colaboradores tengan el servicio de psicología y psiquiatría como parte de los beneficios institucionales pactados.

Uno de los factores más importantes y comunes detectados en la población es el sedentarismo, la falta de acondicionamiento físico del personal y el permanecer sin cambios de postura. Estos hechos han aumentado la presencia de trastorno músculos esqueléticos 
y metabólicos cuya consecuencia podría desencadenar en el aumento de problemas cardiovasculares. Es necesario realizar la evaluación de uso de las herramientas tecnológicas, ya que existe el uso de laptop para trabajos de oficina sin los respectivos acondicionamientos ergonómicos en las instituciones. En algunas ocasiones los trabajadores se ven obligados a llevar trabajo a sus domicilios (tiempo antes de pandemia) y esto incrementaba las horas de trabajo y exposición a factores que pueden influir al riesgo laboral. Los ambientes en los cuales existe asilamiento, alta presencia de humedad y calor son propicios para el aparecimiento de problemas respiratorios y su fácil dispersión.

La sobrecarga de trabajo, la mala utilización de habilidades, la falta de control, el conflicto de autoridad, la desigualdad en el salario, la falta de seguridad en el trabajo, los problemas en las relaciones laborales, el trabajo peligroso físico puedes ser una de las razones más comunes que pueden afectar a la salud de los trabajadores en mediano y largo plazo (OIT, 1984). El ruido y las distracciones en el ambiente son considerados un factor moderadamente nocivo para algunos trabajadores. Las condiciones térmicas del lugar también son una de las características que pueden afectar el desempeño laboral del personal. La sobrecarga de trabajo puede ser cuantitativa (demasiado que hacer) o cualitativa (demasiado difícil). El equilibrio entre los factores humanos y condiciones de trabajo es primordial para el éxito de la interacción del personal en el ambiente de trabajo (OIT, 1984; OIT, 2020; Segura Delgado, 2020).

Una forma eficiente de prevención de riesgos biológicos, es el desarrollo de campañas de inmunización para mitigación de la transmisión de virus respiratorios podría reducir el contagio de la población. Adicionalmente, la inclusión de campañas que fortalezcan la aplicación de medidas higiénicas básicas de prevención de contagio de enfermedades. Se deberán implementar como medidas obligatorias en todas las áreas de las instituciones algunas acciones: el lavado de manos constante del personal para disminuir la transmisibilidad, el uso de mascarillas de protección y ventilación de las oficinas, aulas y espacios compartidos para evaluar posibles mejoras.

Basados en los procesos analizados en la institución, el mayor efecto ambiental en la institución es el impacto por el uso del espacio; que, a través de la planeación, organización, ejecución, control y vigilancia, se podría mejorar. Es responsabilidad de los gestores institucionales, el garantizar sitios de trabajo seguros, sanos y garantes del bienestar físico, mental y social de cada colaborador. Estas acciones mejorarían resultados vinculados al desempeño, desarrollo y productividad dentro de la organización.

Dentro del establecimiento, cualquier aspecto medioambiental (externo) o ambiental laboral (interno) que se mida en las instalaciones, podría, en algún punto, afectar el confort o bienestar de las personas. Cuando se da este caso, debe prioritariamente ser considerado como un aspecto ambiental importante para controlar y prevenir.

La administración de la gestión preventiva sirve para cumplir con la responsabilidad social en la salud de una institución. La dispersión geográfica y el volumen de los trabajadores 
en las organizaciones pueden influir en el aspecto de organización de una institución y en la toma de decisiones sobre la prevención de riesgos.

La sostenibilidad de un entorno laboral debe asentarse sobre la satisfacción de las necesidades de los trabajadores en temas de salud, seguridad en el entorno físico de trabajo, bienestar psicosocial y fortalecimiento de relaciones interpersonales en un entorno feliz, sano y placentero (Marsollier, 2011). Por tal motivo es importante evaluar estos problemas y ver mecanismos para la mitigación de los mismos. Para ello, se debe crear los protocolos específicos para el uso de pantallas de visualización de datos, como parte de las acciones para mitigación y detección temprana de problemas relacionados como son los músculos esqueléticos, neurológicos y oftalmológicos. Se recomienda la creación de procedimientos para la realización de pausas músculos esqueléticos y la generación de programas que promuevan de manera obligatoria esta actividad como parte de la cultura preventiva.

El ausentismo laboral conlleva un gasto presupuestario anual importante en cada institución. El tema depriorizarevaluaciones desalud ocupacionallleva a tenerorganizaciones saludables. Los aspectos de protección, sostenibilidad y salud forman parte de los Objetivos de Desarrollo Sostenible que son metas que todos los gobiernos deben considerar para la mejora continua. La gestión preventiva es la solución para evitar la incidencia de riesgos tanto psicosociales como ergonómicos y a través de la intervención psicosocial se puede desarrollar medidas de prevención (ISST, 2015). Participar en una intervención psicosocial es motivador tanto para el gestor como para los colaboradores y propicia la base para la implicación posterior cuando se deba desarrollar medidas preventivas institucionales.

\section{CONCLUSIONES}

$\mathrm{E}$ s necesario mantener una vigilancia permanente de las condiciones relacionadas a factores psicosociales que son los más difíciles de identificar cuando no se encuentran en estados críticos en la población trabajadora. A través de metodologías fácilmente aplicables y de uso rutinario se podría medir y detectar a tiempo índices sub-estándares críticos.

La planificación y la promoción de campañas y programas que velen por el bienestar del personal, garantizará el sostenimiento de la institución, desempeño de los trabajadores y fortalecerá el compromiso de los mismos en un entorno saludable.

Es necesario fortalecer la comunicación organizacional para que los trabajadores puedan acudir de forma inmediata y oportuna a los primeros síntomas o manifestaciones de disminución de calidad de clima laboral y bienestar al realizar sus funciones en los puestos de trabajo.

Es fundamental evaluar las condiciones estructurales de infraestructura (ventilación, iluminación, ruido ambiental, confort térmico, etc.) y del mobiliario para evitar que se presenten problemas de salud en la población. 
Se debe culturizar a los trabajadores sobre la importancia de tener buenos hábitos de postura, uso de equipos informáticos, uso de mobiliario dentro de la oficina, periodos de recuperación como son pausas activas y recreativas durante la jornada laboral y actividad física y recreacional fuera de la institución como parte del compromiso de mantener un buen estado de salud.

Se debe educar a los trabajadores respecto a buenos hábitos tanto higiénicos sanitarios, manejo correcto de los alimentos, frecuencia de consumo para prevenir patologías gastrointestinales como la gastritis, gastroenteritis y colitis, por lo cual es importante promover programas de salud integral entre varios departamentos institucionales: nutrición, bienestar y centros médicos.

Se recomienda la generación de un proyecto para mejorar el estado físico, bajar el sedentarismo y mejorar las dietas con aumento de consumo en grasas no saturadas; además de reforzar un mejor manejo de trastornos metabólicos, se debe trabajar en un proyecto con nutrición sobre educación alimentaria.

\section{REFERENCIAS}

Alonso, J., Angermeyer, M. C., Bernert, S., Bruffaerts, R., Brugha, T. S., Bryson, H. et al. (2004). Prevalence of mental disorders in Europe: results from the European Study of the Epidemiology of Mental Disorders (ESEMeD) project. Acta Psychiatrica Scandinavica: Supplementum, (420), 21-27

American Psychological Association (2010). Trastornos de la ansiedad: el papel de la psicoterapia en el tratamiento eficaz. Recuperado de https://www.apa.org/ centrodeapoyo/tratamiento

Barra, E. E., Cancino, V., Lagos, G., Leal, P. y San Martin, J. (2005). Factores psicosociales y problemas de la salud reportados por adolescentes. Psicología y Salud, 15(2), 231239.

Gil Monte, y Pedro R. 2014. Manual de psicosociología aplicada al trabajo y a la prevención de los riesgos laborales. Madrid, ESPAÑA: Difusora Larousse - Ediciones Pirámide. Consulta: 22 de septiembre de 2020. En: http://portal.uasb.edu.ec:8888. ProQuest ebrary
Instituto Nacional de Seguridad e Higiene en el Trabajo (INSHT). (2015). Algunas orientaciones para evaluar los factores de riesgo psicosocial (edición ampliada 2015). Recuperado de: https://www. insst.es/documents/94886/96076/ Maqueta+18+4+Angel+lara.pdf/9b38de84a9e0-4c08-bce4-92b5ff4f0861

Marsollier, R. (2011). El Bienestar psicológico en el trabajo y su vinculación con el afrontamiento en situaciones conflictivas. Psicoperspectivas. Individuo y Sociedad, 10(1): 209-220. Recuperado de https:// www.psicoperspectivas.cl/index.php/ psicoperspectivas/article/viewFile/125/131

Organización Internacional del Trabajo (1984). Factores psicosociales en el trabajo: Naturaleza, incidencia y prevención. Serie Seguridad, higiene y medicina del Trabajo. Número 56. Oficina Internacional del Trabajo, Ginebra, Suiza. Recuperado de http://www.factorespsicosociales.com/wpcontent/uploads/2019/02/FPS-OIT-OMS.pdf 
Organización Internacional del Trabajo (2020). Riesgos psicosociales: un reto en materia de seguridad y salud en el trabajo. Recuperado de https://www.ilo.org/Search5/search. do ?searchLanguage=esysearchWhat =riesgos+psicosociales

Organización Mundial de la Salud (2020). ¿Cómo define la OMS la salud? Recuperado de https://www.who.int/es/about/who-we-are/ frequently-asked-questions

Riechmann, J., y Tickner J. (2002). Introducción al principio de la precaución. Barcelona. Icaria. pp. 160

Salanova M. (2008). La psicología de la salud ocupacional positiva- entrevista a marisa salanova. Recuperado de: http://www. infocop.es/view_article.asp?id=1853

Salanova, M., Llorens, S., y Martínez, I.M. (2016). Aportaciones desde la psicología organizacional positiva para desarrollar organizaciones saludables y resilientes. Papeles del Psicólogo, 37(3): 177-184. Recuperadode:http://repositori.uji.es/xmlui/ bitstream handle/10234/164266/74737 _ \% 28 e s p a \% C $3 \%$ B 1 ol \% 29 .pdf ? sequence $=1$ yisAllowed $=y$

Segura Delgado, A. L. (2020). Factores de riesgo psicosocial asociados a la calidad de vida de los trabajadores [Tesis de pregrado, Universidad Cooperativa de Colombia]. Repositorio Institucional UCC. Recuperado de https://repository.ucc.edu. co/handle/20.500.12494/20162

Sociedad Venezolana de Psicología Positiva (2014). Aportes de la psicología positiva al bienestar en el trabajo: aprendizajes de una experiencia venezolana. Caracas, Sociedad Venezolana de Psicología Positiva Recuperado de http://biblioteca.univalle.edu.ni/ files/ original/8c03f37ed7e5884b638ebbfd 558c999c7b191b98.pdf\#page $=12$

Soler, G., Flores, E., Flores, D. y Cortés, J.A. (2019). Manual del modelo pedagógico ITSCO: Fundamentación teórica y científica del modelo de educación superior integrador de emociones y valores orientado a resultados de aprendizaje del Instituto Tecnológico Superior Cordillera (ITSCO) ( $1^{\text {a }}$ ed.). Quito: ITSCO

World Health Organization (2020). Los servicios de salud mental se están viendo perturbados por la COVID-19 en la mayoría de los países, según un estudio de la OMS. https://www. who.int/es/news/item/05-10-2020 -covid19-disrupting-mental-health-services-inmost-countries-who-survey 